\title{
Genetic diversity and evolutionary relationships among Legionella pneumophila clinical isolates, Portugal, 1987 to 2012
}

M J Chasqueira (mjchasqueira@fcm.unl.pt) ${ }^{1}$, L Rodrigues ${ }^{1}$, M Nascimento $^{2}$, M Ramos $^{3}$, T Marques $1,2,4$

1. Faculdade de Ciências Médicas, Universidade Nova de Lisboa, Microbiology Department, CEDOC, Lisboa, Portugal

2. Hospital de Egas Moniz - CHLO, Clinical Microbiology Laboratory, Lisboa, Portugal

3. Direcção Geral de Saúde, Directorate of Analysis and Information, Lisboa, Portugal

4. Coordinator of the Portuguese Legionnaires'Disease Surveillance Programme, Lisboa, Portugal

Citation style for this article:

Chasqueira MJ, Rodrigues L, Nascimento M, Ramos M, Marques T. Genetic diversity and evolutionary relationships among Legionella pneumophila clinical isolates, Portugal, 1987 to 2012. Euro Surveill. 2014;19(46):pii=20965. Available online: http://www.eurosurveillance.org/ViewArticle.aspx?Articleld=20965

Article submitted on 31 October 2013 / published on 20 November 2014

The genetic diversity of 89 clinical Legionella isolates, collected between 1987 and 2012, in 22 hospitals from the five regions of Portugal, was analysed in this study using monoclonal antibodies (MAbs) of the Dresden panel and the sequence-based typing (SBT) protocol. The eBURST algorithm was used to infer levels of relatedness between isolates. All isolates collected were Legionella pneumophila, which were further characterised into four subgroups by MAbs, and 30 sequence types (STs) by SBT. Twelve of the STs were unique to Portugal; one of them (ST100) was represented by 32 epidemiologically related isolates. The ST44 was the profile with the highest number of epidemiologically unrelated isolates. The eBURST analyses indicate that, within the group formed by the 30 STs identified in this study, 17 STs were genetically close to at least another ST in the group. The comparison between the eBURST diagrams obtained with the STs from this study and the entire SBT database of the European Working Group for Legionella, showed that 24 (seven of them unique to Portugal) of our 30 STs were related with STs identified in others countries. These results suggest that the population of $L$. pneumophila clinical strains in Portugal includes both worldwide and local strains.

\section{Introduction}

Legionellaceae are ubiquitous in the environment, being particularly prevalent in man-made habitats, such as cooling towers and domestic hot and cold water distribution systems. This family consists of a single genus, Legionella, but contains 56 species/subspecies belonging to over 70 serogroups [1]. Legionella are the causative agents of Legionnaires' disease (LD), a severe pneumonia that is transmitted through inhalation of contaminated aerosols. The most common species to cause disease is L. pneumophila, which has 16 serogroups, but the majority of human disease $(84 \%$ worldwide, $95 \%$ in Europe) is caused by L. pneumophila serogroup (sg) $1[2,3]$.

When a case of LD occurs, it is essential that public health authorities are able to detect the source of infection promptly by comparing clinical and environmental isolates, so that decontamination measures can prevent further cases. For this comparison, the sequencebased typing (SBT) scheme and monoclonal antibodies (MAbs) of the Dresden panel are the typing methods widely used by the members of the European Working Group for Legionella Infections (EWGLI), renamed to European Study Group for Legionella Infections (ESGLIESCMID), since September 2012.

In 1999, LD was included in the Portuguese system of mandatory notifications of infectious diseases. In $\mathbf{2 0 0 4}$, an integrated programme of epidemiological surveillance was implemented in order to improve reporting, diagnosis and investigation of cases, through the inclusion of obligatory laboratory notification and communication of guidelines to the professionals involved.

In Portugal the number of reported cases per year between 2004 and 2012 ranged from 61 to 140. The peak in the number of cases is usually between August and November and most of the notifications come from the North region. From 2004 to 2012, a total of 868 cases were notified (crude annual reporting rate of $0.91 / 100,000$ inhabitants) with 50 fatalities (case fatality ratio of $6 \%)$. The majority of cases $(672 / 868,77 \%)$ were male and the most affected age groups were the 50 to 59 (217 cases, 25\%) and 40 to 49 year-olds (196 cases, $23 \%$ ) [4].

As partner of this surveillance scheme, the Legionella Laboratory of the Microbiology Department of the Faculdade de Ciências Médicas (Lisbon) is responsible for the characterisation of the clinical strains isolated 
in Portugal. In a previous study, the L. pneumophila clinical strains isolated in Portugal were characterised by MAbs and SBT methodologies, which allowed the construction of a database for use in epidemiological surveillance efforts [5].

The aim of the current study was to assess the genetic diversity and evolutionary relationships among $L$. pneumophila clinical isolates collected in Portugal during the period from 1987 to 2012, and to compare these results with the available data submitted to EWGLI-SBT database until 10 April 2013.

\section{Methods}

\section{Origin and epidemiological characteristics of} isolates

In total, 89 clinical Legionella isolates were analysed. Forty-one clinical isolates were obtained from the 868 cases reported to the integrated programme of epidemiological surveillance and 48 clinical isolates came from the collection of the Microbiology Laboratory of Hospital Egas Moniz-CHLO (1987 to 2003). These 89 clinical isolates, all from patients requiring hospitalisation, were sent by 22 hospitals from five regions of Portugal: 60 from Lisbon, 25 from the North, two from the Centre, one from Alentejo and one from Algarve. Thirty-six of the 60 strains from Lisbon were isolated at the same hospital (hospital A) between 1987 and 2007.

Among the 89 total isolates, 36 of the isolates were collected from patients with nosocomial infections and 39 from community-acquired infections. The remaining 14 isolates had an unknown origin. All but one of the nosocomial isolates came from hospital $A$; the other strain was isolated during a small outbreak in hospital $B$ in the North region. Only five of the isolates from community-acquired infections were related cases and came from two outbreaks, one in 2009 and the other in 2012, in two cities of the North region of Portugal.

The median ages of patients ( 63 males and 16 females; 10 unknown) were 55 and 58 years-old for males and females, respectively (range: $13-80$ years; 16 patients with unknown age).

\section{Phenotype, genotype and diversity of isolates}

The L. pneumophila strains were phenotyped at the Microbiology Laboratory of Hospital Egas MonizCHLO with MAbs of the Dresden panel [6]. Genotyping was performed at the Legionella Laboratory of the Microbiology Department of the Faculdade de Ciências Médicas using a seven-allele standard SBT scheme [7-9]. Briefly, the Dresden panel identifies 15 of the 16 L. pneumophila sgs using sg-specific MAbs. In addition, for the sg1 of L. pneumophila, the Dresden panel uses five MAbs plus the Mab 3 of the International Panel obtained from the American Type Culture Collection [10]. With this scheme of six MAbs, it is possible to differentiate the sg1 strains into nine subgroups (Philadelphia,
Allentown/France, Knoxville, Olda, Benidorm, Oxford, Bellingham, Heysham and Camperdown) [6]. Both panels were performed by direct immunofluorescence and are based on the reactivity of the surface lipopolysaccharide epitopes.

Within the SBT scheme, part of the genes flaA, pilE, asd, mip, mompS, proA, and neuA, were sequenced. For each gene sequence a distinct allele number is assigned through the EWGLI-SBT database for L. pneumophila (available at: http://www.hpa-bioinformatics. org.uk/legionella/legionella_sbt/php/sbt_homepage. php). The combination of seven allele numbers defines an allelic profile to which a sequence type (ST) is attributed. In order to assign a ST, when the standard neuA primers failed to amplify, we used the novel primer set, specifically designed for the neuA homolog (neuAh) [11]. This neuAh is present in some non-sg1 strains and is functionally equivalent to the neuA gene of the strain Philadelphia-1 [12].

Clinical isolates diversity was assessed by calculating Hunter and Gaston's modification of Simpson's index of diversity (IOD) [13], using the V-Dice application (available at: http://www.hpa-bioinformatics.org.uk/ cgi-bin/DICl/DICI.pl).

\section{Assessing relationships between isolates}

For establishing the possible evolutionary relationships between isolates, we applied the eBURST algorithm v3 (available at: http://eburst.mlst.net). The eBURST group was obtained with a less stringent definition, in which STs are included within the same group only if they share identical alleles at five or six of the seven SBT loci with at least one other ST. STs that cannot be assigned to any group are called singletons. The statistical confidences for the primary founders were assessed using 1,000 bootstrap re-samplings [14].

\section{Results}

\section{Characteristics and diversity of isolates}

All the 89 clinical isolates were L. pneumophila and 84 belonged to $\mathrm{sg} 1$, the other five were serotyped as sg8, 10, 12 and 14 . Of the 84 sg1 isolates, 83 had the virulence-associated epitope recognised by $M \mathrm{Mb}_{3} / 1$; 33 belonged to the Philadelphia subgroup, 30 to the Allentown/France, 17 to the Knoxville and three to the Benidorm. The only isolate $M \mathrm{Mb}_{3} / 1$ negative was characterised as OLDA. The Knoxville was the most widespread subgroup in our country, being present in four of five regions investigated (North, Centre, Lisbon and Algarve).

With regard to the category of the infection, the strains from community-acquired cases showed greater diversity (four subgroups: Philadelphia, Allentown/France, Knoxville and Benidorm) than the nosocomial cases (two subgroups: Philadelphia and Allentown/France). 
Sequence-based typing of clinical Legionella pneumophila isolates, all MAb3/1 positive, from 22 hospitals located in five regions $^{\mathrm{a}}$ of Portugal, 1987-2012 ( $\left.\mathrm{n}=83\right)$

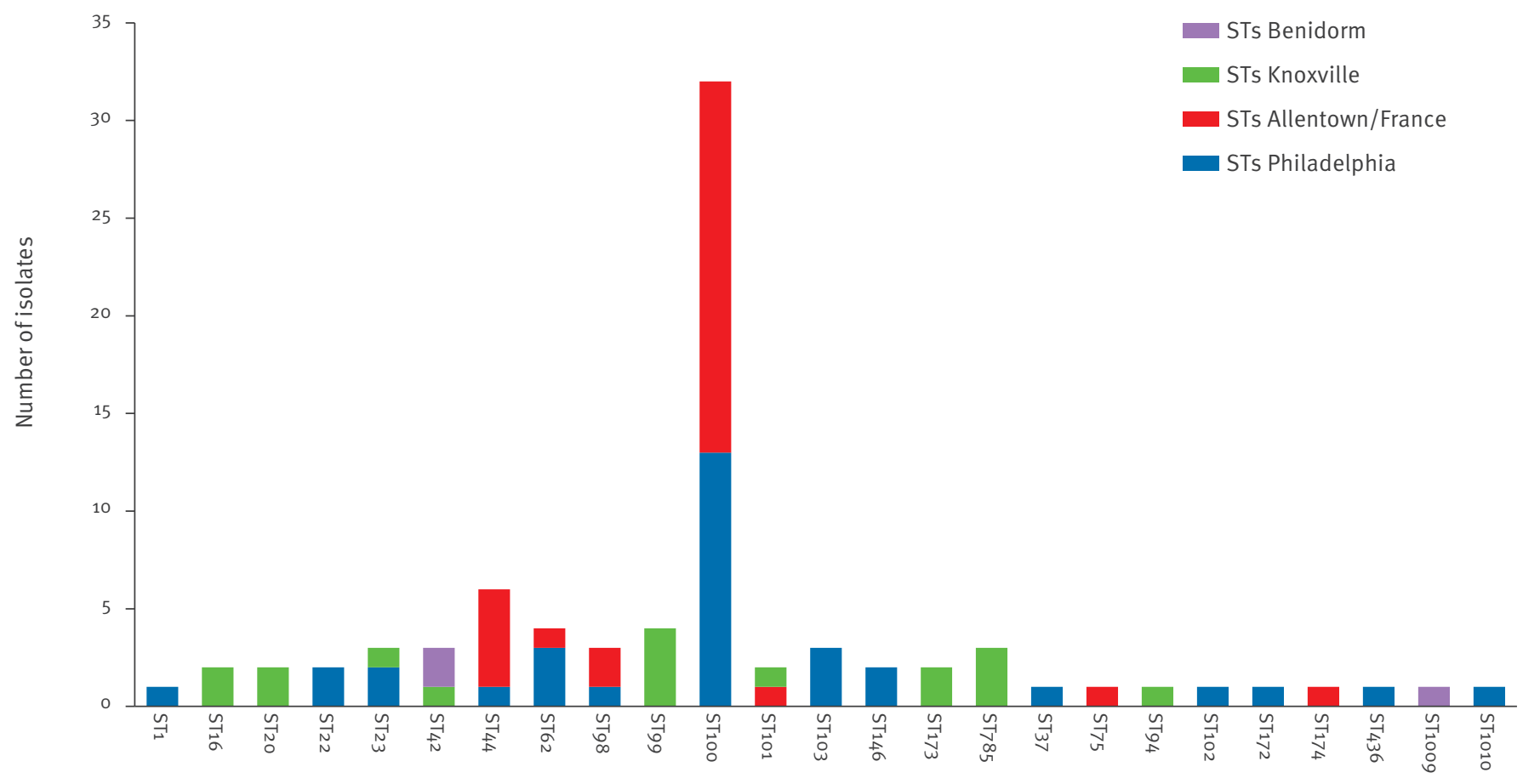

SBT profile

SBT: sequence-based typing; ST: sequence type.

a The five regions were: Lisbon, the North, the Centre, Alentejo and Algarve.

All the strains from the sg1 and one from sg12 were genotyped by standard SBT. Figure 1 shows the repartition per ST of the 83 isolates positive for $M A b_{3} / 1$, as well as the sg1 subgroups within each ST. The neuA primers failed to amplify four isolates, all of them nonsg1 $(\mathrm{sg} 8,10,14)$. As described in the methods section, in these cases we used the neuAh primers, which improved the quality of the sequences and allowed us to assign a ST to these isolates.

Of the 89 L. pneumophila clinical isolates available for SBT analysis, 52 were from single clinical cases (unrelated strains, comprising the 14 of unknown origin), the remaining isolates were associated to outbreaks (related strains: five strains were recovered from the two community outbreaks in 2009 and 2012, and 32 were isolated in hospital A from nosocomial infections). After inclusion of one isolate for each group of epidemiologically related strains, 55 isolates (these three isolates plus the 52 unrelated strains) were included in our SBT analysis. These 55 isolates were found to include $30 \mathrm{STs}(\mathrm{IOD}=0.972 ; 95 \%$ confidence interval (CI): 0.960-0.985), with the mompS gene being the most discriminative $(I O D=0.893)$ and the gene proA the less discriminative $(I O D=0.748)$.

Fourteen STs consisted of groups containing between two and five unrelated isolates and the remaining STs accounted for only one single isolate each. Twelve of the 30 STs were unique to Portugal, according to data submitted to the EWGLI-SBT database at the time of writing: nine STs from L. pneumophila sg1 (ST98, ST100, ST101, ST102, ST173, ST174, ST785, ST1009, ST1010) and three STs from L. pneumophila non-sg1 (ST1343, ST1383 and ST1384). Altogether, these new STs comprised 16 of the 55 isolates included in this study. In addition, eight new allele numbers were assigned by the EWGLI-SBT database curators after our data were submitted to the database ( 22 and 29 for the mip gene, 24 and 37 for the pilE gene and 20, 34, 23 and 219 for the asd, mompS, proA and neuAh genes, respectively). It is interesting to note that six of these new allele numbers were detected only in L. pneumophila non-sg1 strains.

The ST100, one of the profiles exclusively found in our country, was restricted to hospital A, with 32 isolates. Another ST specific to our country was the ST785; this profile, which was represented by three isolates in our study, was only found in the 2009 community outbreak involving eight cases in the North region.

The ST44, found in five strains, was the profile with the highest number of clinical unrelated isolates. All of them were recovered from community-acquired infections. This ST was also associated with the community 
eBURST analysis of Legionella pneumophila sequence types obtained from clinical isolates, Portugal, 1987-2012

A. Relationships between the 30 L. pneumophila sequence types observed in Portugal during the study

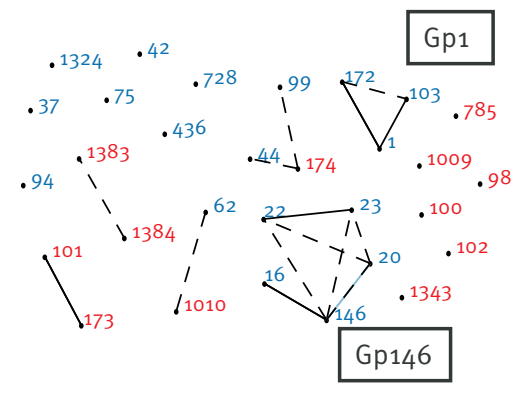

B, C. Two of the 24 clonal complexes obtained by applying the eBURST algorithm on 1,451 sequence types reported to the EWGLI-SBT database

B

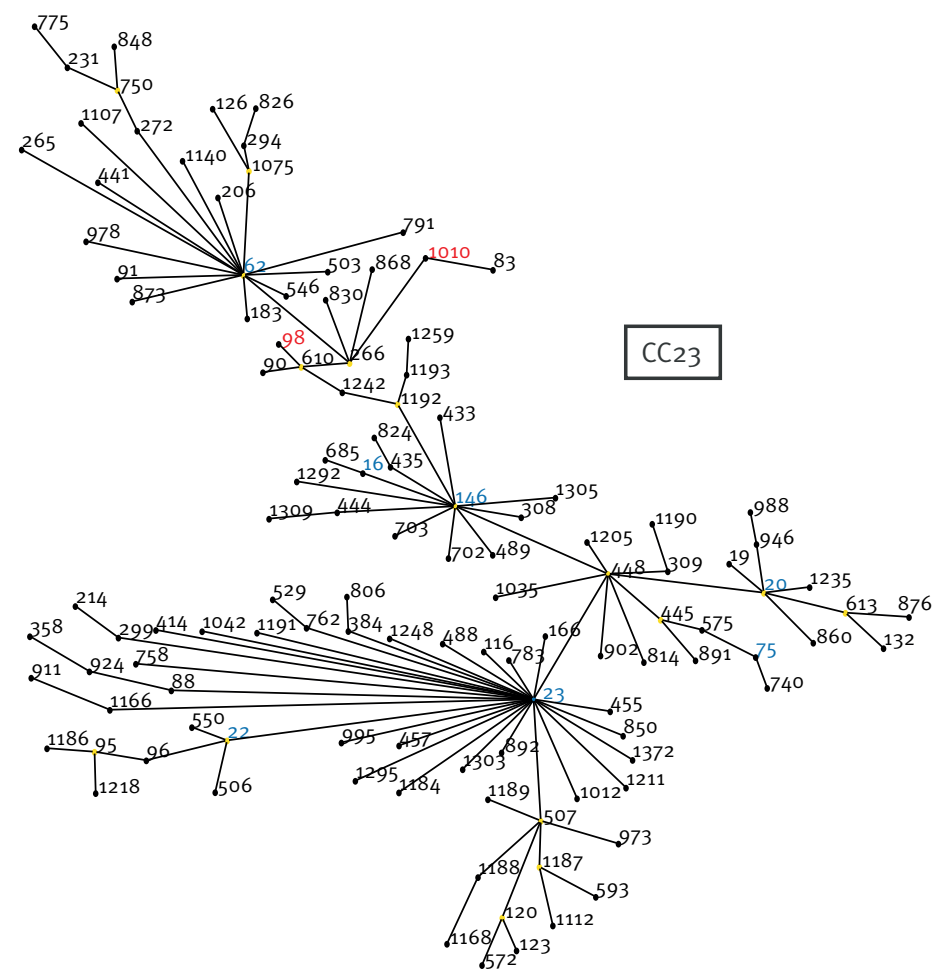

C

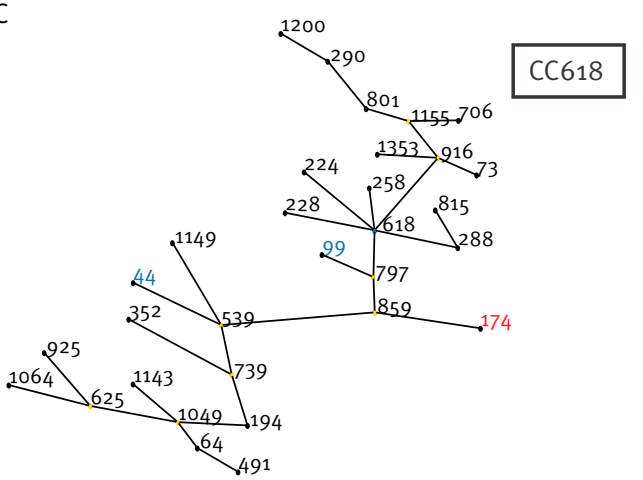

CC: clonal complex; EWGLI-SBT: European Working Group for Legionella Infections-sequence-based typing.

In red were the STs unique to Portugal and in blue the STs from Portugal, which can also be found in other countries. Full lines link singlelocus variants and broken lines link double-locus variants. Blue spots represent founders and yellow spots represent sub-founders.

outbreak in 2012, in the North region, from which two isolates were analysed here.

The ST62 and ST99 were found in four clinical isolates each. Two of the ST62 strains were associated with nosocomial infections, one recovered from a single case in hospital A, in 1990; and the other was associated with a small outbreak, involving three cases, in hospital B, in 2011. Two other STs, ST172 and ST1384, were also detected in single isolates from hospital $A$. The ST99 was retrieved, between 2000 and 2009, from community-acquired infections in the North region, Centre and Algarve.
The ST1 was found only twice among our strains, and both isolates were obtained from sporadic cases in 1995, in the Lisbon region.

The combination of the two methodologies, MAbs and SBT, showed that the Philadelphia subgroup was the most heterogeneous as it was divided into 14 different STs (Figure 1).

\section{Evolutionary relationships among isolates}

The 30 STs generated in this study were subdivided by eBURST into six groups (Gp) and 13 singletons using the less stringent definition (Figure $2 \mathrm{~A}$ ). The six groups include 17 STs and 38 of the 55 unrelated clinical isolates studied. The Gp146 was the most representative 
group, containing five STs (ST16, ST20, ST22, ST23 and $\mathrm{ST}_{146)}$ and 11 isolates, all of them recovered from sporadic cases of community-acquired infections. The ST146 was the hypothetic founder of the group, from which derived one single-locus variant (SLV) and three double-locus variants (DLVs). The ST146 comprised only two strains, isolated in 2007 and 2008, in the North region. The $\mathrm{Gp}_{1}$ is composed by $\mathrm{ST}_{1}$, the predicted founder of the group, from which derived two SLVs, ST103 and ST172. All of the six strains included in this group were isolated in the Lisbon region between 1991 and 1999.

All but two of the 13 singletons STs were represented by a single strain each; the ST42 and ST98 were associated with three strains each.

According to the results of our eBURST analysis, six of the twelve STs unique to Portugal were singletons (ST98, ST100, ST102, ST785, ST1009 and ST1343). Of the remaining STs found exclusively in our country, the ST101 and ST173 were related, diverging only in neuA. The ST1383 and ST1384 were slightly more distant from each other, being DLV. The ST174 was a DLV with ST44 and ST99, which were the STs with highest number of clinical unrelated isolates in this study.

Using the eBURST algorithm on the entire EWGLI-SBT database, 24 clonal complexes (CC) were generated. These CCs were obtained with the most stringent definition, i.e. all the STs are SLVs. With this method, 24 of the STs from our country were assigned into eight of these CC. The other six STs cannot be linked with any other ST of the database, being singletons (ST100, ST436, ST785, ST1343, ST1383 and ST1384).

The $\mathrm{CC}_{59}$ (ST59 was the predicted founder with bootstrap support of $100 \%$ ), included three STs identified in Portugal (ST101, ST173 and ST728). Five STs that circulated in our country (ST1, ST37, ST103, ST172 and ST1009) were associated to $\mathrm{CC}_{1}$. Two of these STs (ST37 and ST1009) were singletons in the first eBURST analysis. The CC23 included our ST62, ST1010, Gp146 (that enclosed ST16, ST20, ST22, ST23 and ST146), and two other STs (ST75 and ST98), that in the previous eBURST diagram were singletons (Figure $2 \mathrm{~A}$ and Figure $2 \mathrm{~B}$ ). In this CC the ST62, ST146, and ST20, emerged as subfounders (bootstrap subgroup value of $100 \%, 98 \%$ and $79 \%$, respectively). These STs diversified from the founder and generated their own SLVs (i.e. had at least two links to other STs previously unassigned, and the link to the progenitor). Two of the most represented STs in our collection (ST44 and ST99) were grouped in CC618 (Figure 2C).

An overview of the eBURST diagram using the entire EWGLI-SBT database showed that nine of 13 ST that were singletons in the first eBURST analysis were now associated with a CC; among these, it should be highlighted that the ST42 and ST94 were predicted founders of two CCs. Five of the 12 STs specific to Portugal were assigned as singletons in this analysis (ST100, $\mathrm{ST}_{7}$, $, \mathrm{ST} 1343, \mathrm{ST} 1383$ and ST1384).

\section{Discussion}

In Portugal, like other countries, most of the diagnoses of LD are currently made by antigen detection in urine, due to the simplicity, rapidity and specificity of this test for L. pneumophila sg1. Culture is not a methodology widely used and, therefore, Legionella clinical isolates are not available for the majority of LD cases, which limits epidemiological studies $[15,16]$. Actually, this was observed in the current study, since only 41 clinical isolates were obtained from 868 cases reported to the integrated programme of epidemiological surveillance.

Another limitation of the present study is that our population of isolates represents the more severe cases of disease, as all isolates were from patients requiring hospitalisation. Therefore, the present report may not entirely reflect the distribution of the Legionella strains responsible for LD in Portugal, because cases with less severe disease are probably underrepresented in this collection. However, this study probably provides a good representation of the circulating STs in the country.

During the 25-year study period, we gathered 89 clinical isolates, 55 of them from unrelated cases. The majority of the clinical isolates $(n=63)$ were obtained from men, which is in accordance with the literature [17-19].

As expected, the majority $(n=84)$ of the clinical isolates were L. pneumophila sg1. Using the MAbs of Dresden panel all but one of the strains possessed virulence-associated epitope recognised by $M A b_{3} / 1$; this is in line with previous reports suggesting that these strains are more likely to cause disease than the others not exhibiting this phenotype $[6,17]$. In our study, the two major subgroups were Philadelphia and Allentown/France. These two MAb patterns only differ in the MAb8/4 reactivity and we had verified that, using indirect immunofluorescence in some strains, the MAb8/4 staining was heterogenic, with only 1 to $2 \%$ of the bacteria showing a strong positive signal; in these cases, we classified them as Allentown/France.

The SBT results showed significant genetic diversity, which is in accordance with reports from other countries. The diversity of clinical isolates in our study (0.972) was similar to that respectively described previously in Japan (0.979) [17], Canada (0.964) [20], England and Wales (0.901) [21], and slightly greater than in Belgium (0.879) [18]. As in other studies, momps proved to be the most discriminating locus [22].

In this study, 12 of the 30 STs detected were new to the EWGLI-SBT database. These results suggest that the population of L. pneumophila clinical strains circulating 
in Portugal, during the last 25 years, was a combination of worldwide and local strains.

The STs of the 89 isolates analysed were uploaded to the EWGLI-SBT database, which allows comparison between countries. Over the study period, the ST44 was linked with the highest number of Portuguese clinical unrelated isolates. According to the EWGLI-SBT database, the ST44 has repeatedly been associated with clinical isolates from France, Germany, Italy, and United Kingdom, with the first one recovered in 1994. The other major STs were ST62 and ST99, both with four clinical isolates. The EWGLI-SBT data show that ST62 is found ubiquitously across Europe but ST99 was isolated only once in the Brussels region, in 2008.

The ST1 appears in the EWGLI-SBT database as the profile most frequently reported around the world, with 1,132 of the 8,300 strains reported. Interestingly, one of the strains associated with this profile was isolated in 1947, in Washington, from a patient with pneumonia during an outbreak of unknown aetiology. The reanalysis of this unclassified agent in 1979, by McDade, showed that it was the same species as the LD bacterium [23].

This report provides the evolutionary relationships among the L. pneumophila clinical isolates collected in Portugal during the period from 1987 to 2012 by using the eBURST algorithm. This algorithm uses STs to divide bacterial populations into groups of closely related strains based on the theory that a genotype within a population starts diversifying, by mutation or recombination, to produce variants that differ in only one or two of the seven loci.

In the eBURST analysis of STs detected in this study, the less stringent definition for groups was chosen due to the small number of isolates tested $(n=89)$. The data generated indicate that 13 STs do not relate with any other ST, and for the remaining, nine were SLVs and 11 were DLVs. Thus, the STs involved in these links are considered to have diverged very recently.

In the diagram drawn with the 1,451 STs reported to the EWGLI-SBT database, eBURST identified four of the STs detected in Portugal (ST1, ST23, ST42 and ST94) as founder genotypes of CCs. Another six STs (ST20, ST22, ST37, ST62, ST146 and ST172) were also predicted as subgroup founders. This means that from these STs many other genotypes have diversified.

In conclusion, we applied SBT and the eBURST algorithm to examine the genetic diversity and evolutionary relationships among the $L$. pneumophila clinical isolates collected in Portugal during the period from 1987 to 2012. This study provides information about the strains circulating in Portugal and their relationship with strains from other countries. However, additional research is required in order to improve the knowledge of the geographic distribution of virulent clones.
Acknowledgments

This study was supported by the Portuguese Foundation for Science and Technology (FCT) [grant number PTDC/ SAU-ESA/64269/2006].

Conflicts of interest

None declared.

\section{Authors's contribution}

Maria-Jesus Chasqueira: designed the study and the analytical strategy, final approval of the version. Lúcia Rodrigues: implementation of molecular biology technique; interpretation of data; critical review of the manuscript; final approval of the version. Marta Nascimento: implementation of phenotypic technique; final approval of the version. Marina Ramos: analysis of data from the integrated programme of epidemiological surveillance; final approval of the version. Teresa Marques: critical review of the manuscript; final approval of the version.

\section{References}

1. Gomez-Valero L, Rusniok C, Buchrieser C. Legionella pneumophila: population genetics, phylogeny and genomics. Infect Genet Evol. 2009;9(5):727-39. http://dx.doi. org/10.1016/j.meegid.2009.05.004 PMID:19450709

2. Yu VL, Plouffe JF, Pastoris MC, Stout JE, Schousboe M, Widmer $A$, et al. Distribution of Legionella species and serogroups isolated by culture in patients with sporadic communityacquired legionellosis: an international collaborative survey. J Infect Dis. 2002;186(1):127-8. http://dx.doi. org/10.1086/341087 PMID:12089674

3. Harrison TG, Doshi N, Fry NK, Joseph CA. Comparison of clinical and environmental isolates of Legionella pneumophila obtained in the UK over 19 years. Clin Microbiol Infect. 2007;13(1):78-85. http://dx.doi.org/10.1111/j.14690691.2006.01558.x PMID:17184291

4. Direcção-Geral da Saúde. Programa de Vigilância Epidemiológica Integrada da Doença dos Legionários: Notificação Clínica e Laboratorial de Casos. Base de dados 2004 - 2012. [Directorate-General of Health. National Epidemiological Surveillance Network. Database from 2004 to 2012]. Lisbon: Direcção-Geral da Saúde. Portuguese.

5. Chasqueira MJ, Rodrigues L, Nascimento M, Marques T. Sequence-based and monoclonal antibody typing of Legionella pneumophila isolated from patients in Portugal during 19872008. Euro Surveill. 2009;14(28):pii=19271. PMID:19607780

6. Helbig JH, Bernander S, Castellani Pastoris M, Etienne J, Gaia V, Lauwers S, et al. Pan-European study on culture-proven Legionnaires' disease: distribution of Legionella pneumophila serogroups and monoclonal subgroups. Eur J Clin Microbiol Infect Dis. 2002;21(10):710-6. http://dx.doi.org/10.1007/ S10096-002-0820-3 PMID:12415469

7. Gaia V, Fry NK, Afshar B, Lück PC, Meugnier H, Etienne J, et al. Consensus sequence-based scheme for epidemiological typing of clinical and environmental isolates of Legionella pneumophila. J Clin Microbiol. 2005;43(5):2047-52. http:// dx.doi.org/10.1128/JCM.43.5.2047-2052.2005 PMID:15872220

8. Ratzow S, Gaia V, Helbig JH, Fry NK, Lück PC. Addition of neuA, the gene encoding $\mathrm{N}$-acylneuraminate cytidylyl transferase, increases the discriminatory ability of the consensus sequence-based scheme for typing Legionella pneumophila serogroup 1 strains. J Clin Microbiol. 2007;45(6):1965-8. http:// dx.doi.org/10.1128/JCM.00261-07 PMID:17409215

9. Mentasti M, Fry NK. ESCMID Study Group for Legionella Infections (ESGLI) Sequence-Based Typing protocol for epidemiological typing of Legionella pneumophila Version 5.0, October 2012;pp 1-9. Basel: ESGLI. Available from: http:// www.hpa-bioinformatics.org.uk/legionella/legionella_sbt/ php/sbt_homepage.php

10. Joly JR, McKinney RM, Tobin JO, Bibb WF, Watkins ID, Ramsay D. Development of a standardized subgrouping scheme for Legionella pneumophila serogroup 1 using monoclonal antibodies. J Clin Microbiol. 1986;23(4):768-71. PMID: 3517064

11. Mentasti M, Fry NK. ESCMID Study Group for Legionella Infections (ESGLI). Analysis of the N-Acylneuraminate 
Cytidyltransferase homologue (neuAh) found in some nonserogroup 1 strains of Legionella pneumophila. Version 1.0, October 2012. Basel: ESGLI. Available from: http://www. hpa-bioinformatics.org.uk/legionella/legionella_sbt/php/ sbt_homepage.php

12. Farhat C, Mentasti M, Jacobs E, Fry NK, Lück C. The $\mathrm{N}$-acylneuraminate cytidyltransferase gene, neuA, is heterogenous in Legionella pneumophila strains but can be used as a marker for epidemiological typing in the consensus sequence-based typing scheme. J Clin Microbiol. 2011;49(12):4052-8. http://dx.doi.org/10.1128/JCM.00687-11 PMID:21956982

13. Hunter PR, Gaston MA. Numerical index of the discriminatory ability of typing systems: an application of Simpson's index of diversity. J Clin Microbiol. 1988;26(11):2465-6. PMID:3069867

14. Feil EJ, Li BC, Aanensen DM, Hanage WP, Spratt BG. eBURST: inferring patterns of evolutionary descent among clusters of related bacterial genotypes from multilocus sequence typing data. J Bacteriol. 2004;186(5):1518-30. http://dx.doi. org/10.1128/JB.186.5.1518-1530.2004 PMID:14973027

15. Joseph CA, Ricketts KD, Yadav R, Patel S; European Working Group for Legionella Infections. Travel-associated Legionnaires disease in Europe in 2009. Euro Surveill. 2010;15(41):pii=19683. PMID:20961516

16. Beauté J, Zucs P, de Jong B; European Legionnaires' Disease Surveillance Network. Legionnaires disease in Europe, 20092010. Euro Surveill. 2013;18(10): pii=20417. PMID:23515061

17. Amemura-Maekawa J, Kura F, Helbig JH, Chang B, Kaneko A, Watanabe Y, et al.; Working Group for Legionella in Japan. Characterization of Legionella pneumophila isolates from patients in Japan according to serogroups, monoclonal antibody subgroups and sequence types. J Med Microbiol. 2010;59(Pt 6):653-9. http://dx.doi.org/10.1099/jmm.0.017509o PMID:20185546

18. Vekens E, Soetens O, De Mendonça R, Echahidi F, Roisin S, Deplano A, et al. Sequence-based typing of Legionella pneumophila serogroup 1 clinical isolates from Belgium between 2000 and 2010. Euro Surveill. 2012;17(43):pii=20302. PMID: 23137466

19. Vanaclocha H, Guiral S, Morera V, Calatayud MA, Castellanos M, Moya V, et al. Preliminary report: outbreak of Legionnaires disease in a hotel in Calp, Spain, update on 22 February 2012 Euro Surveill. 2012;17(8):pii=20093. PMID:22401506

20. Reimer AR, Au S, Schindle S, Bernard KA. Legionella pneumophila monoclonal antibody subgroups and DNA sequence types isolated in Canada between 1981 and 2009: Laboratory Component of National Surveillance. Eur J Clin Microbiol Infect Dis. 2010;29(2):191-205. http://dx.doi. org/10.1007/s10096-009-0840-3 PMID:19960359

21. Harrison TG, Afshar B, Doshi N, Fry NK, Lee JV. Distribution of Legionella pneumophila serogroups, monoclonal antibody subgroups and DNA sequence types in recent clinical and environmental isolates from England and Wales (2000-2008). Eur J Clin Microbiol Infect Dis. 2009;28(7):781-91. http://dx.doi. org/10.1007/s10096-009-0705-9 PMID:19156453

22. Edwards MT, Fry NK, Harrison TG. Clonal population structure of Legionella pneumophila inferred from allelic profiling. Microbiology. 2008;154(Pt 3):852-64. http://dx.doi. org/10.1099/mic.0.2007/012336-o PMID:18310031

23. McDade JE, Brenner DJ, Bozeman FM. Legionnaires' disease bacterium isolated in 1947. Ann Intern Med. 1979;90(4):659-61. http://dx.doi.org/10.7326/0003-4819-90-4-659 PMID:373548 